\title{
Combined structural and diffusion tensor imaging detection of ischemic injury in moyamoya disease: relation to disease advancement and cerebral hypoperfusion
}

\author{
Ken Kazumata, MD, PhD, ${ }^{1}$ Kikutaro Tokairin, MD, ${ }^{1}$ Masaki Ito, MD, PhD, ${ }^{1}$ Haruto Uchino, MD, PhD, ${ }^{1}$ \\ Taku Sugiyama, MD, PhD, ${ }^{1}$ Masahito Kawabori, MD, PhD, ${ }^{1}$ Toshiya Osanai, MD, PhD,1 \\ Khin Khin Tha, MD, PhD, ${ }^{2}$ and Kiyohiro Houkin, MD, PhD'
}

1Department of Neurosurgery, Hokkaido University Graduate School of Medicine; and ${ }^{2} \mathrm{Clinical}$ Research and Medical Innovation Center, Hokkaido University Hospital, Sapporo, Japan

OBJECTIVE The microstructural integrity of gray and white matter is decreased in adult moyamoya disease, suggesting covert ischemic injury as a mechanism of cognitive dysfunction. Establishing a microstructural brain imaging marker is critical for monitoring cognitive outcomes following surgical interventions. The authors of the present study determined the pathophysiological basis of altered microstructural brain injury in relation to advanced arterial occlusion, cerebral hypoperfusion, and cognitive function.

METHODS The authors examined 58 patients without apparent brain lesions and 30 healthy controls by using structural $\mathrm{MRI}$, as well as diffusion tensor imaging (DTI). Arterial occlusion in each hemisphere was classified as early or advanced stage based on MRA and posterior cerebral artery (PCA) involvement. Regional cerebral blood flow (rCBF) was measured with $N$-isopropyl- $p$-[123]]-iodoamphetamine SPECT. Furthermore, cognitive performance was examined using the Wechsler Adult Intelligence Scale, Third Edition and the Trail Making Test (TMT). Both voxel- and region of interest-based analyses were performed for groupwise comparisons, as well as correlation analysis, using parameters such as cognitive test scores; gray matter volume; fractional anisotropy (FA) of association fiber tracts, including the inferior frontooccipital fasciculus (IFOF) and superior longitudinal fasciculus (SLF); PCA involvement; and rCBF.

RESULTS Compared to the early stages, advanced stages of arterial occlusion in the left hemisphere were associated with a lower Performance IQ $(p=0.031)$, decreased anterior cingulate volumes $(p=0.0001$, uncorrected), and lower $F A$ in the IFOF, cingulum, and forceps major (all $p<0.01$, all uncorrected). There was no significant difference in rCBF between the early and the advanced stage. In patients with an advanced stage, PCA involvement was correlated with a significantly lower Full Scale IQ $(p=0.036)$, cingulate volume $(p<0.01$, uncorrected), and FA of the left $\operatorname{SLF}(p=0.0002$, uncorrected) compared to those with an intact PCA. The rCBF was positively correlated with FA of the SLF, IFOF, and forceps major $(r>0.34, p<0.05)$. Global gray matter volumes were moderately correlated with TMT part $A(r=0.40, p=$ 0.003). FA values in the left SLF were moderately associated with processing speed $(r=0.40, p=0.002)$.

CONCLUSIONS Although hemodynamic compensation may mask cerebral ischemia in advanced stages of adult moyamoya disease, the disease progression is detrimental to gray and white matter microstructure as well as cognition. In particular, additional PCA involvement in advanced disease stages may impair key neural substrates such as the cingulum and SLF. Thus, combined structural MRI and DTI are potentially useful for tracking the neural integrity of key neural substrates associated with cognitive function and detecting subtle anatomical changes associated with persistent ischemia, as well as disease progression.

https://thejns.org/doi/abs/10.3171/2020.1.JNS193260

KEYWORDS cerebral blood flow; cognitive disorder; diffusion tensor imaging; moyamoya disease; volumetric magnetic resonance imaging; vascular disorders

\footnotetext{
ABBREVIATIONS CBF = cerebral blood flow; DTI = diffusion tensor imaging; FA = fractional anisotropy; FIQ = Full Scale IQ; IFOF = inferior frontooccipital fasciculus; ILF = inferior longitudinal fasciculus; $\mathrm{MCA}=$ middle cerebral artery; $\mathrm{PCA}=$ posterior cerebral artery; $\mathrm{PIQ}=$ Performance $\mathrm{IQ} ; \mathrm{PO}=$ Perceptual Organization; $\mathrm{PS}=\mathrm{Processing}$ Speed; $r C B F=$ regional $C B F ; R O I=$ region of interest; $S L F=$ superior longitudinal fasciculus; $T B S S=$ tract-based spatial statistics; TFCE = threshold-free cluster enhancement; TMT = Trail Making Test; VBM = voxel-based morphometry; VC = Verbal Comprehension; VIQ = Verbal IQ; WAIS-III = Wechsler Adult Intelligence Scale, Third Edition; WM = Working Memory.
}

SUBMITTED December 2, 2019. ACCEPTED January 20, 2020

INCLUDE WHEN CITING Published online April 3, 2020; DOI: 10.3171/2020.1.JNS193260. 
$\mathrm{M}$ OyAmOya disease, a rare cerebrovascular disorder, involves noninflammatory occlusive changes in the intracranial internal carotid artery. A state of chronic hypoperfusion is considered to interfere with the normal development of a child's brain, as well as with executive function (dysexecutive disorder) in adults with moyamoya disease..$^{1,2}$ Recently, the evaluation of microstructural damage to the white matter has garnered attention in the study of moyamoya disease. ${ }^{3-6}$ Diffusion tensor imaging (DTI) markers for subtle white matter damage may reveal the ischemic burden and its progression; thus, the metrics can serve as a measure beyond the assessment of cerebral blood flow (CBF) and metabolism. Furthermore, measurements of the brain microstructure can be used to evaluate the effects of therapeutic interventions such as revascularization surgery. ${ }^{7-9}$

Diffusion imaging, postacquisition image processing techniques, and quantification of diffusion parameters allow one to determine the profile of white matter injuries such as reduced fiber density, demyelination, and axonal injury..$^{10}$ In moyamoya disease, DTI parameters such as fractional anisotropy (FA), radial diffusivity, and mean diffusivity are altered. ${ }^{5}$ Diffusion kurtosis imaging demonstrates reduced tissue complexity in the fiber-crossing white matter region of moyamoya patients compared to that of healthy subjects. ${ }^{4}$ Neurite orientation dispersion and density imaging in patients with moyamoya reveal a lower intracellular volume fraction in comparison with that in healthy subjects. ${ }^{3}$ Detecting subtle damage to the brain in patients without apparent brain lesions is also performed by measuring gray matter volumes. A previous study revealed focal atrophy in the posterior cingulum in patients with moyamoya disease. ${ }^{5}$ Although the results of some studies have suggested that chronic ischemia reduces gray and white matter integrity, the effects of cortical hypoperfusion are still unclear., 3,5 Advanced arterial involvement, as well as posterior cerebral artery (PCA) occlusion, is observed during the course of moyamoya disease. Cerebral ischemia is often masked because of hemodynamic compensation in adult moyamoya disease. Furthermore, PCA involvement exhausts the source of collateral blood flow; however, conventional imaging modalities do not always demonstrate reduced cortical perfusion. ${ }^{11}$ Nevertheless, an association between disease advancement, including PCA occlusion, and cognitive impairment has been suggested..$^{12,13}$ However, the impact of disease advancement on brain microstructure, as well as cognitive function, remains unclear.

Thus, in the present study, we aimed to examine the association between MRI-derived parameters of microstructural brain damage and cognitive function, as well as MRA findings and regional $\mathrm{CBF}(\mathrm{rCBF})$ measurements. We investigated the effects of arterial lesion progression with a focus on the impact of any PCA involvement and subsequent $\mathrm{rCBF}$ changes in the brain microstructure. We also examined the correlation between MRI-derived indexes of the brain microstructure and cognitive performance parameters to determine efficient structural markers for brain function assessment in moyamoya disease.

\section{Methods}

This study was approved by the Research Ethics Com- mittee of Hokkaido University Hospital. Informed consent was obtained from all patients. The protocols adhered to the principles set forth in the US Code of Federal Regulations, Title 45, Public Welfare, Part 46, Protection of Human Subjects, revised January 15, 2009, and the World Medical Association Declaration of Helsinki.

\section{Participants}

Consecutive patients (age $\geq 18$ years) diagnosed with moyamoya disease at our hospital between November 2012 and March 2018 were included in this prospective, observational, single-center study. All patients fulfilled criteria for the diagnosis of moyamoya disease set by the Research Committee on Moyamoya Disease established by the Ministry of Health, Labour and Welfare of Japan. ${ }^{14}$ Exclusion criteria were apparent cortical/subcortical infarction on conventional MRI, intracranial hemorrhage, revascularization surgery before the study, neurological deficit due to stroke, and comorbid illnesses that could affect cognition. The present study was performed as an extension of our related work and includes 23 patients previously analyzed. ${ }^{5}$ A total of 58 adult patients were included in the present study (mean age $40.5 \pm 9.8$ years [mean \pm standard deviation], range 21-58 years; female/male ratio 41:17). Inclusion criteria for controls were as follows: no clinical evidence of psychiatric or neurological disorders; IQ in the normal range, as assessed by the Japanese version of the National Adult Reading Test (JART); no brain lesions on conventional MRI; and no current medication that could affect cognitive function. The control group consisted of 30 participants (mean age $38.1 \pm 7.6$ years, range $27-56$ years; female/male ratio 16:14). The mean estimated IQ of the control group was $105.8 \pm 7.6$.

\section{Conventional Radiological and Cognitive Examinations}

All patients underwent MRI and MRA. The MRI was performed on a 3.0-T imaging unit (Achieva TX, Philips Medical Solutions). The severity of occlusive lesions from the internal carotid artery to the middle cerebral artery (MCA) was assessed with MRA. We classified the lesions into two categories: early stage, normal or mild to moderate stenosis when the majority of the distal branches were visible; and advanced stage, severe stenosis or occlusion when the majority of the distal branches were not visible. The advanced stage in the MRA-based classification corresponds to stage 3 or above in Suzuki's classification. ${ }^{15}$ We also evaluated the PCA lesions using MRA.

Intellectual ability was assessed using the Wechsler Adult Intelligence Scale, Third Edition (WAIS-III). In addition to the Full Scale IQ (FIQ), four underlying index scores were assessed: Verbal Comprehension (VC), Perceptual Organization (PO), Working Memory (WM), and Processing Speed (PS). Executive function was evaluated using the Trail Making Test (TMT; parts A and B). In the TMT, z-transformed scores were generated using ageappropriate normative values. Administrators of cognitive tests were blinded to the patient clinical information.

\section{CBF Measurements}

The rCBF values were measured using $N$-isopropyl- 
$p$-[123I]-iodoamphetamine SPECT (IMP-SPECT) with a scanner (GCA-9300R, Toshiba Medical Systems Corp.). Images were normalized to the standard template of the Statistical Parametric Mapping version 12 (SPM12; Wellcome Center for Human Neuroimaging, www.fil.ion.ucl. ac.uk/spm/) ${ }^{16}$ Region of interest (ROI) analysis was performed to extract rCBF values from images in the standardized space. Templates of ROIs were generated using the ATTbasedFlowTerritories atlas that covers MCA perfusion territories (Fig. 1) ${ }^{17}$ The rCBF values for each cerebellar hemisphere were also extracted, and the averages of the right and the left values were used for normalization of the $\mathrm{rCBF}$.

\section{Measurements of Brain Microstructure Using Structural and Diffusion MRI}

To evaluate subtle gray and white matter alterations, 3D magnetization-prepared rapid gradient-echo T1-weighted imaging (3D-MPRAGE) and axial single-shot spin-echo echo-planar DTI were performed. The scan parameters for DTI were as follows: TR $5051 \mathrm{msec}$, TE $85 \mathrm{msec}$, flip angle $90^{\circ}$, FOV $224 \times 224 \mathrm{~mm}^{2}$, matrix size $128 \times 128$, b-value $1000 \mathrm{sec} / \mathrm{mm}^{2}$, number of diffusion gradient directions 32 , slice thickness $3 \mathrm{~mm}$, number of slices 43 , and number of excitations 1 . The 3D-MPRAGE was performed with TR $6.8 \mathrm{msec}$, TE $3.1 \mathrm{msec}$, flip angle $8^{\circ}$, and TI 1100 msec.

Voxel-based morphometry (VBM) was conducted using SPM12 running on MATLAB version R2015b (MathWorks). MR images were segmented into gray matter, white matter, and cerebrospinal fluid using the standard segmentation module. ${ }^{18}$ Diffeomorphic Anatomical Registration using Exponentiated Lie Algebra (DARTEL) was used to spatially normalize the gray matter segments to the DARTEL template supplied with the VBM8 toolbox (http://dbm.neuro.uni-jena.de/vbm). ${ }^{19}$ After initial affine registration of the DARTEL template to the gray matter tissue probability map in the Montreal Neurological Institute (MNI) space (http://www.mni.mcgill.ca/), nonlinear warping of the segmented images was performed to match the MNI space DARTEL template. Gray matter images were modulated and smoothed using an 8-mm full width at half maximum (FWHM) isotropic gaussian kernel. Both the spatially normalized and modulated gray matter data were used for voxel- and ROI-based analysis (Fig. 1). In addition to measuring the global gray matter volume, ROI analysis was performed to extract regional gray matter volume values from the images in standardized space using automated anatomical labeling. ${ }^{20}$

The diffusion imaging data were processed using the FSL library (http://fsl.fmrib.ox.ac.uk/fsl/fslwiki/). ${ }^{21}$ The DTI indexes of FA data were extracted using FSL with the default parameters. Each subject's FA images were normalized using the FLIRT (FMRIB's Linear Image Registration Tool) algorithm in FSL. Alterations in diffusion parameters were compared voxel-wise using tract-based spatial statistics (TBSS) implemented in FSL. In TBSS, voxel-wise permutation-based nonparametric inferences were determined using skeletonized FA data with the FSL Randomise function. An ROI-based analysis was performed to extract FA values from the images in the standardized space. Anatomical ROIs were defined using the definitions in the JHU white matter tractography atlas, which defines 20 structures available in FSL version 5.0.10. ${ }^{22}$ ROIs were placed in the anterior thalamic radiation, cingulum (four segments), forceps major/minor, inferior frontooccipital fasciculus (IFOF), inferior longitudinal fasciculus (ILF), and superior longitudinal fasciculus (SLF; two segments; Fig. 1).

\section{Statistical Analyses}

Voxel-wise group comparisons were performed using VBM and TBSS to identify regions showing microstructural gray and white matter differences between the patients and controls (Fig. 2). Voxel-wise correlation analyses were also performed with VBM and TBSS to test the effect of gray matter volume and white matter integrity on cognitive performance, using age as a nuisance covariate. In VBM, the statistical significance level was set as $\mathrm{p}<$ 0.001 without multiple comparison corrections. In TBSS, statistical significance was tested with 1000 permutations and a threshold set at $\mathrm{p}<0.05$, correcting for multiple comparisons by means of threshold-free cluster enhancement (TFCE).

In the ROI-based analysis, group comparisons between controls and patients were performed both in 90 regional gray matter volumes and in 20 white matter tracts. The analysis yielded 44 gray matter regions, as well as 13 white matter tracts, which demonstrated statistically significant differences after multiple comparisons with Bonferroni correction. Subsequently, differences in gray matter volume, FA, and $\mathrm{rCBF}$ in relation to the MRA presentations (early or advanced stage) were investigated by analysis of covariance using age as a covariate to adjust for differences and effects. Cognitive test scores were also compared using the unpaired t-test for patients with and without advanced stage of arterial involvement. The additional effect of PCA involvement was further analyzed in patients showing an advanced stage of arterial involvement, comparing cognitive test performances, $\mathrm{rCBF}$, gray matter volume, and FA. In the ROI-based analysis, statistical significance was described with uncorrected $\mathrm{p}$ values. Pearson's correlation coefficients were used to identify brain structures showing strong correlations with rCBF or cognitive performance.

All statistical analyses except for VBM and TBSS were performed using R (R Foundation for Statistical Computing, http://www.R-project.org/).

\section{Results \\ Clinical Characteristics}

Among the 58 moyamoya patients, 23 demonstrated transient ischemic attack (TIA), whereas the remaining 35 were classified as asymptomatic. Their average intelligence (FIQ, $96.1 \pm 16.7)$, PS $(98.1 \pm 16.9)$, and executive function (TMT part A, $0.07 \pm 0.41$; TMT part B, $0.18 \pm$ 0.50 ; z-score) parameters were within normal range. Demographic and clinical characteristics of the patients are summarized in Table 1.

Our data confirmed substantially lower white matter FA values in moyamoya patients than in controls $(\mathrm{p}<0.05$, 


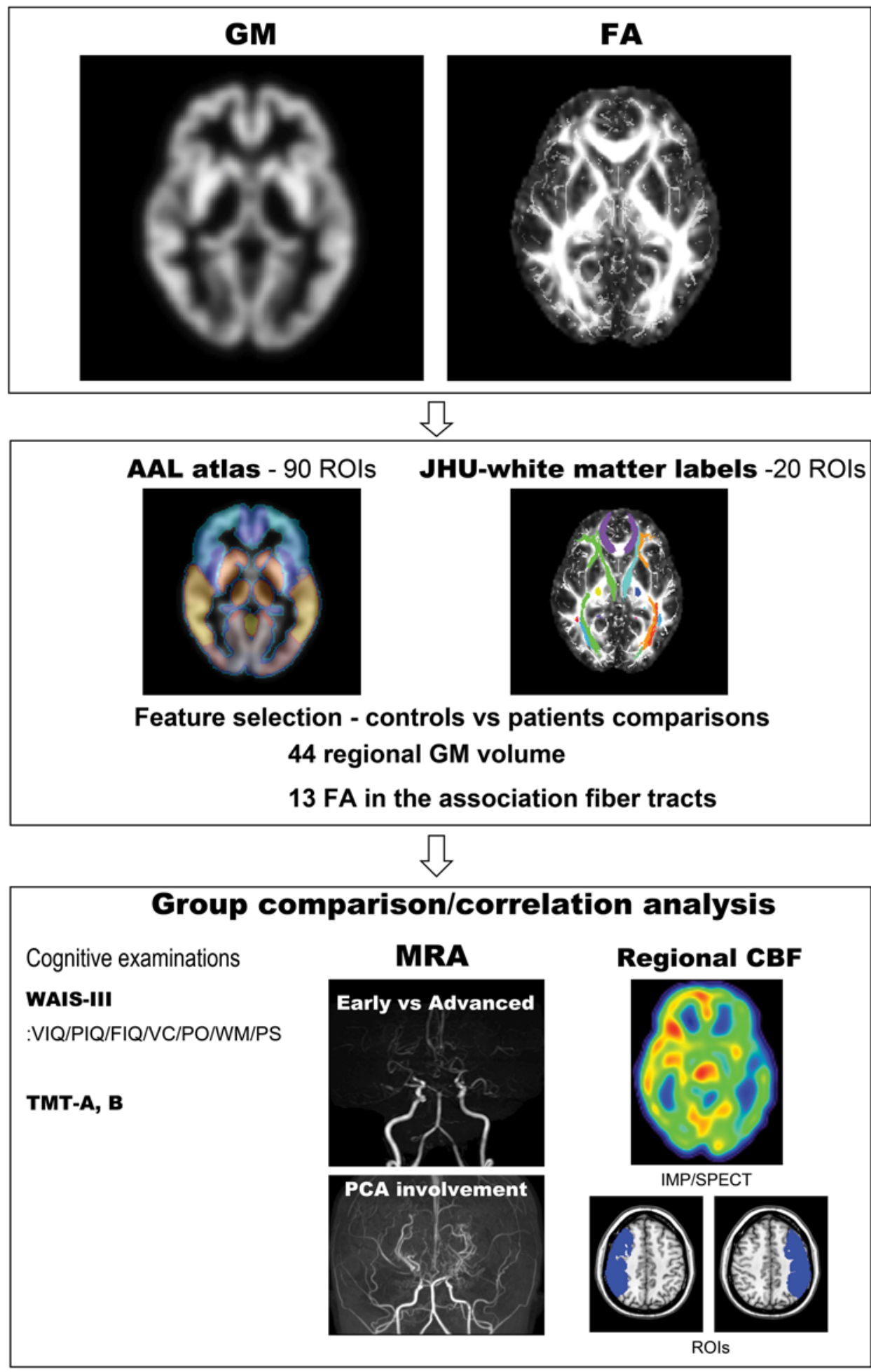

FIG. 1. Flowchart describing the group comparison/correlation analysis. Upper: Gray matter (GM) volume was determined using T1-weighted imaging, whereas the white matter diffusion index of FA was measured using DTI. Center: Regional GM volumes were measured in 90 regions by applying the automated anatomical labeling $(A A L)$ atlas. FA values were extracted from 20 white matter tracts by applying the JHU white matter tractography atlas. Lower: Selected imaging features were used to explore possible relationships among cognitive tests, arterial involvement on MRA, and rCBF. IMP/SPECT $=N$-isopropyl- $p$-[123|]-iodoamphetamine SPECT. 
GM

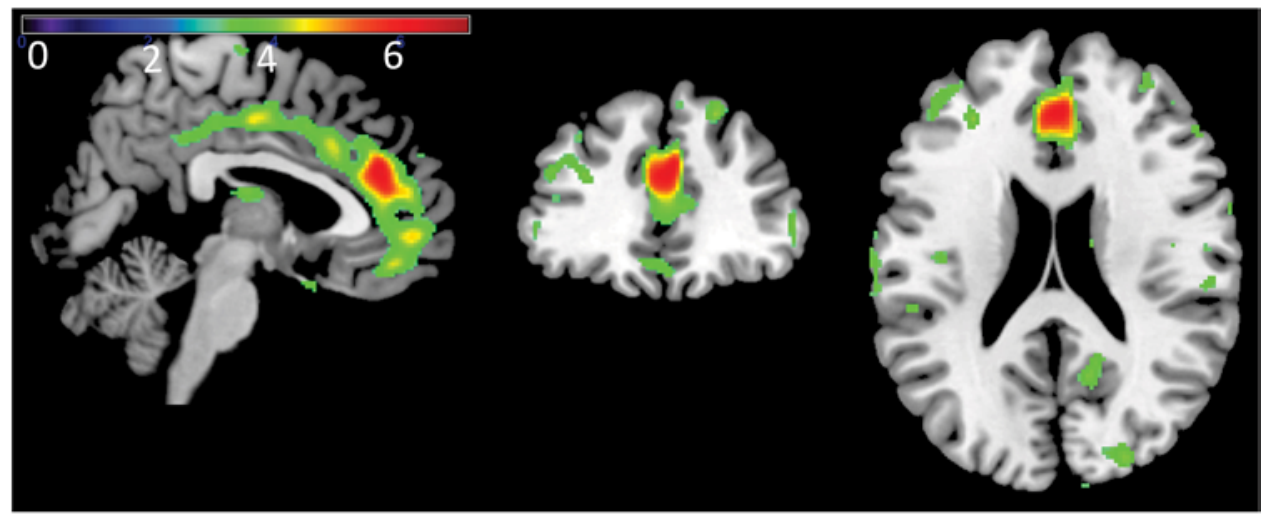

FA

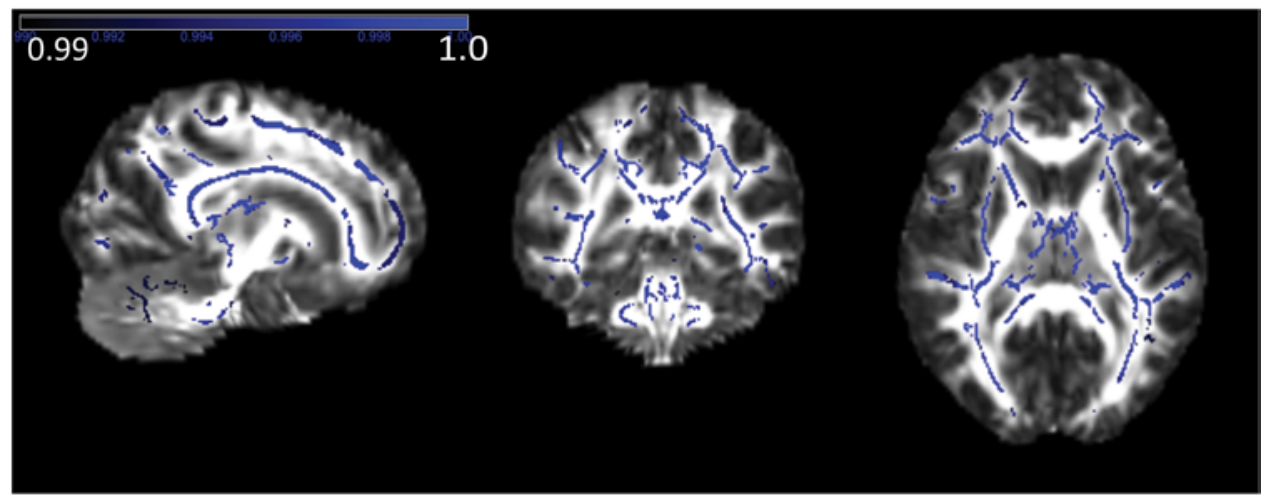

\section{Control vs MMD}

FIG. 2. Upper: The voxel-based group comparison revealed a distinct reduction in the GM volume of the cingulate gyrus $(p<$ 0.001, uncorrected). Lower: The group comparison based on TBSS showed lower white matter FA values $(p<0.05$, TFCE-corrected) in moyamoya patients than in control subjects. The color scale of the GM volume represents t-values, whereas that in the FA panel displays $1-p$ values. MMD = moyamoya disease.

TFCE-corrected; Fig. 2). Although previous studies have revealed lower gray matter volumes mainly in the posterior cingulum, the present study showed that the gray matter volume reduction was strongest in the anterior portion of the cingulum ( $\mathrm{p}<0.001$, uncorrected).

\section{Impact of Arterial Occlusion}

In both hemispheres, there was no statistical difference in $\mathrm{rCBF}$ between early and advanced stages. Advanced arterial occlusion in the left hemisphere was associated with lower Performance IQ (PIQ; early vs advanced, $100.5 \pm$ 13.9 vs $92.1 \pm 14.9, \mathrm{p}=0.031$, two-tailed) and $\mathrm{PO}$ (early vs advanced, $101.3 \pm 14.7$ vs $93.7 \pm 16.1, \mathrm{p}=0.030$, onetailed; Fig. 3A). Patients with advanced arterial involvement in the left hemisphere showed lower performance on TMT part A than patients with early-stage involvement $(-0.02 \pm 0.52$ vs $0.16 \pm 0.19$, respectively, $p=0.0498$, onetailed). By contrast, advanced arterial occlusion in the right hemisphere was not associated with cognitive performance measures.

Advanced arterial occlusion in the left hemisphere was also associated with lower ipsilateral regional gray matter volume such as in the anterior and middle cingula, amygdala, inferior occipital lobe, and pallidum ( $p<0.001$, uncorrected; Fig. 3B), whereas advanced arterial occlusion in the right hemisphere was not associated with gray matter volumes. Moreover, advanced arterial occlusion was associated with lower FA values of the bilateral IFOF, cingulum, and forceps major and the left ILF ( $p<0.01$, uncorrected) than those in early-stage occlusion (Fig. 3C).

Additionally, we analyzed the effects of PCA involvement in patients at an advanced stage. PCA involvement showed a lower $\mathrm{rCBF}$ in the left hemisphere $(0.83 \pm 0.10 \mathrm{vs}$ $0.76 \pm 0.08, p=0.030$, one-tailed test; Fig. $4 \mathrm{~A}$ ). It was also associated with lower values for Verbal IQ (VIQ), PIQ, FIQ, VC, and PS (VIQ, $p=0.0247$; FIQ, $p=0.036$; VC, $p$ $=0.006$; PS, $p=0.032$; all two-tailed; Fig. 4B). Moreover, PCA involvement was associated with a lower global gray matter volume $(\mathrm{p}=0.003)$, as well as decreased regional volumes in the cingulum (left middle, $p=0.0018$; left anterior, $\mathrm{p}=0.003$; right anterior, $\mathrm{p}=0.004$; all uncorrected; Fig. 4C) and the precentral gyrus (right, $p=0.0036$; left, $\mathrm{p}=0.0035$; both uncorrected). Furthermore, PCA involvement was correlated with lower FA values in the left SLF, 
TABLE 1. Summary of study cohort characteristics

\begin{tabular}{|c|c|c|}
\hline Variable & Control & Patients \\
\hline No. & 30 & 58 \\
\hline Age in yrs (SD) & $38.1(7.6)$ & $40.5(9.8)$ \\
\hline Sex: F/M & $16 / 14$ & $41 / 17$ \\
\hline Risk factor (HT, DM, HL), no. & 0 & 7 \\
\hline Body mass index (SD) & - & $23.4(4.4)$ \\
\hline VIQ (SD) & - & $96.4(17.5)$ \\
\hline $\mathrm{PIQ}(\mathrm{SD})$ & - & $96.1(14.9)$ \\
\hline $\mathrm{FIQ}(\mathrm{SD})$ & $105.8(7.6)$ & $96.0(16.7)$ \\
\hline VC (SD) & - & $96.8(17.0)$ \\
\hline $\mathrm{PO}(\mathrm{SD})$ & - & $97.3(15.8)$ \\
\hline WM (SD) & - & $93.2(15.3)$ \\
\hline PS (SD) & - & $98.1(16.9)$ \\
\hline TMT-A z-score (SD) & - & $0.07(0.41)$ \\
\hline TMT-B z-score (SD) & - & $0.18(0.50)$ \\
\hline $\begin{array}{l}\text { Advanced arterial occlusion, rt hemi- } \\
\text { sphere, no. }(\%)\end{array}$ & - & $38(65.5)$ \\
\hline $\begin{array}{l}\text { Advanced arterial occlusion, It hemi- } \\
\text { sphere, no. }(\%)\end{array}$ & - & $28(51.1)$ \\
\hline PCA involvement, no. (\%) & - & $9(15.5)$ \\
\hline
\end{tabular}

$-=$ data not available; $\mathrm{DM}=$ diabetes mellitus; $\mathrm{HL}=$ hyperlipidemia; $\mathrm{HT}=$ hypertension; TMT-A = TMT part A; TMT-B = TMT part B .

left ILF, cingulum, right ILF, and left anterior thalamic radiation ( $p<0.003$, uncorrected; Fig. 4D).

\section{Correlation With rCBF}

There was no significant correlation between cognitive performances and rCBF. There was a weak positive correlation between $\mathrm{rCBF}$ and gray matter volume in the left middle cingulum $(\mathrm{r}=0.28, \mathrm{p}=0.042$, uncorrected). The scatterplots in Fig. 5 demonstrate the correlations between $\mathrm{rCBF}$ and white matter tracts identified in the forceps major, IFOF, and SLF $(r>0.34, p<0.05)$.

\section{Correlations Between MRI-Derived Metrics and Cognitive Performances}

There was a moderate positive correlation between TMT part A performance and global gray matter volume $(r=0.40, p=0.003)$, as well as regional gray matter volumes in multiple brain regions (Fig. 6A). PS was weakly correlated with FA values of the forceps major $(r=0.30$, $\mathrm{p}=0.043$ ), left IFOF $(\mathrm{r}=0.29, \mathrm{p}=0.026)$, ILF (right, $\mathrm{r}=$ $0.30, p=0.020 ;$ left, $r=0.30, p=0.043)$, and right SLF ( $r$ $=0.34, \mathrm{p}=0.009)$ and was moderately correlated with the left SLF $(r=0.40, p=0.002$; Fig. 6B).

\section{Discussion}

In moyamoya disease, the associations of MRI-derived microstructural measurements with cognitive function, disease severity, and cerebral perfusion have not been comprehensively investigated. Cingulate atrophy, as well as a decrease in FA in multiple white matter tracts, is a hallmark of ischemic injury in the normal-appearing brain

\section{A. Cognitive function}

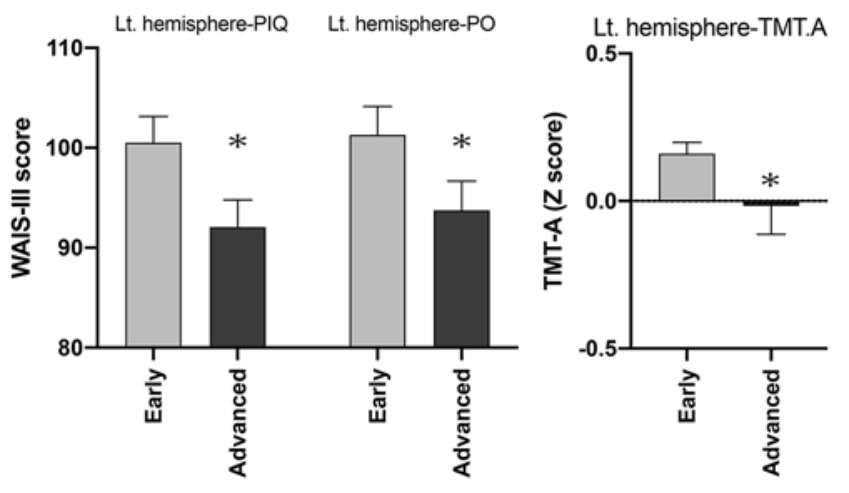

\section{B. Gray matter volume}

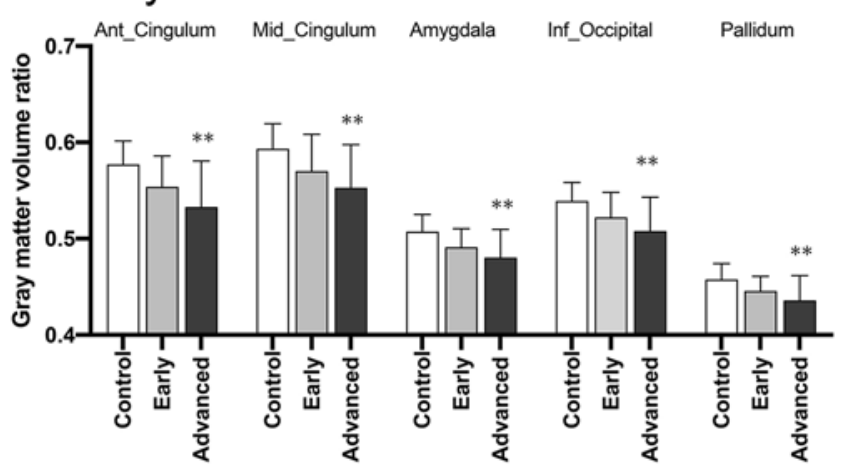

C. White matter integrity

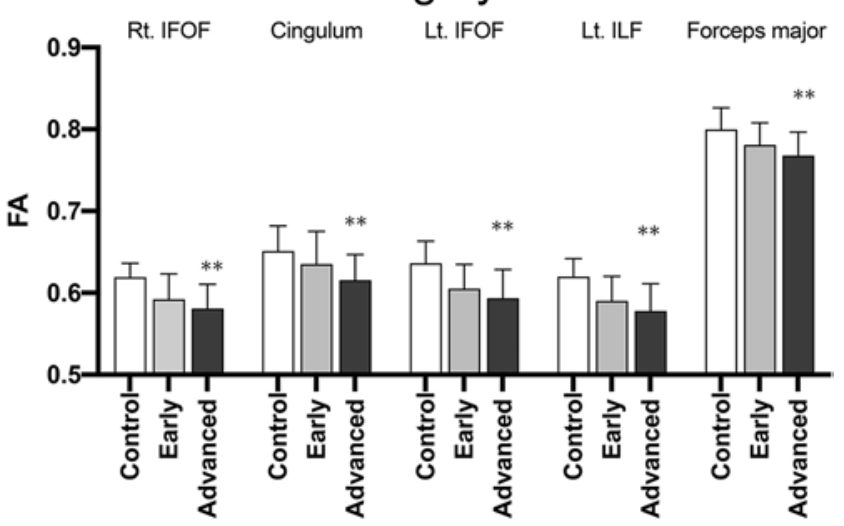

FIG. 3. Arterial involvement stages influence functional and anatomical parameters. A: Box plots indicating cognitive functions evaluated by WAIS-III indexes (PIQ, PO) and the TMT part A. B: At an advanced stage, GM volumes of the left hemisphere are significantly decreased such as in the cingulum (anterior cingulum [Ant_Cingulum], middle cingulum [Mid_Cingulum]), amygdala, inferior occipital lobe (Inf_Occipital), and pallidum. C: In advanced stages, the FA is significantly decreased in white matter tracts such as in the right (Rt.) and left (Lt.) IFOF, left ILF, cingulum, and forceps major. Statistical significance is indicated by asterisks $\left({ }^{*} p<0.05,{ }^{* *} p<0.01\right)$. Analysis of covariance was performed to test the effects of advanced arterial involvement compared with controls, as well as early arterial involvement, including age as a nuisance covariate.

of adults with moyamoya disease. The present study demonstrated that advanced arterial involvement was associated with lower cognitive performances, brain atrophy, and a decrease in white matter integrity. A particular impact on 
A. Regional cerebral blood flow

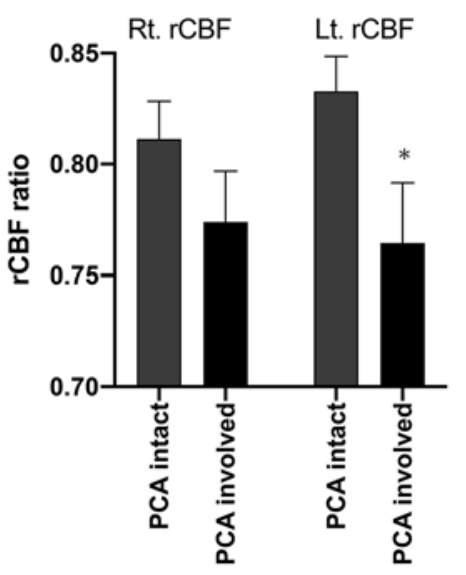

B. Cognitive function

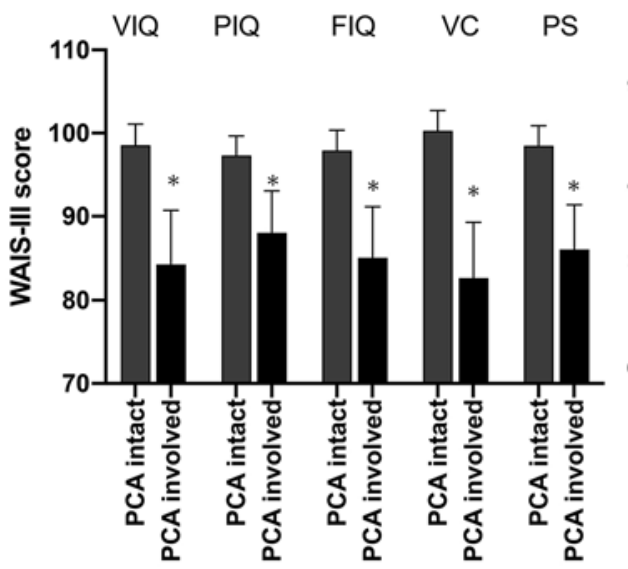

C. Gray matter volume

D. White matter integrity

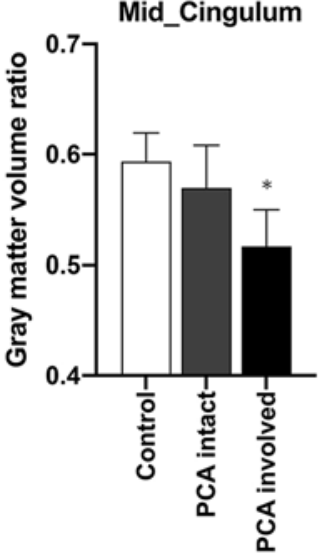

Lt. SLF

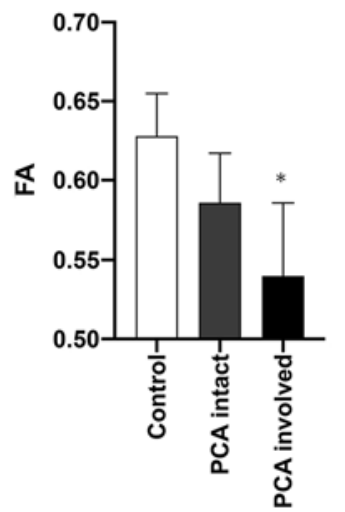

FIG. 4. Regional $\mathrm{CBF}$, cognitive function, GM volume, and white matter integrity in patients with or without PCA involvement.

A: Box plots indicating rCBF with (PCA involved) or without (PCA intact) PCA involvement. Only the left hemisphere showed a significant reduction in rCBF. B: Box plots indicating lower scores in the WAIS-III indexes in patients with PCA involvement compared to those in patients with an intact PCA. C: The GM volume of the left middle cingulum (Mid_Cingulum) is significantly decreased in patients with PCA involvement ( $p=0.002$, uncorrected). D: In patients with PCA involvement, FA of the left (Lt.) SLF is significantly decreased $\left(p=0.0002\right.$, uncorrected). Statistical significance is indicated by asterisks ( $\left.{ }^{*} p<0.01\right)$. Analysis of covariance was performed to test the effect of PCA involvement compared with controls, as well as the advanced stage without PCA involvement, including age as a nuisance covariate.

cognitive performances had PCA involvement characterized by significant cingulate atrophy, as well as reduced white matter integrity in the main association fibers such as the SLF. Furthermore, executive function (TMT) was associated with gray matter volume, whereas PS was associated with white matter integrity. Depending on the severity of arterial involvement and cerebral hypoperfusion, combined structural imaging and DTI may detect subtle ischemic brain injuries that correlate with cognitive functions.

In the present study, we investigated nonsurgically treated patients who lacked apparent cortical/subcortical lesions. We did not find any obvious deviation from the normative values in either intelligence or executive function tests. Similar results were also identified in children with moyamoya disease, ${ }^{23}$ suggesting that changes in cognitive function are modest when the apparent brain lesions are absent in these patients. However, since microstructural alterations have been consistently identified in both gray and white matter in previous studies, ${ }^{3-6}$ their clinical implications need to be clarified in more detail. We speculated that disease progression, as well as cerebral hypoperfusion, is associated with microstructural alterations. Particularly in adult patients, severe impairment of multiple arteries often does not accord with changes in cerebral hemodynamic parameters because hemodynamic compensation mechanisms mask the cerebral hypoperfusion. Therefore, the effects of disease progression could be different from those of cerebral hypoperfusion in terms of the profile of cognitive impairments and the profile of structural changes. Furthermore, a better understanding of the impact of disease progression on cognition and brain structure may alter the treatment strategies, which currently rely heavily on stroke prevention based on CBF and metabolism findings.

In our study, advanced arterial involvement exhibited signs of microstructural injury in the normal-appearing brain. Patients with an advanced stage in the left hemisphere showed a modest decline in the Performance IQ, as well as PO, without significant changes in CBF. The spatial distribution of gray matter atrophy signs suggests the vulnerability of the limbic system. Although moyamoya disease typically demonstrates prefrontal ischemia, the lateral prefrontal region did not present significant atrophy.

PCA involvement is one of the hallmarks of an advanced disease stage. Previous studies have demonstrated that PCA involvement is detected in approximately $11 \%$ of patients after revascularization surgery ${ }^{11}$ and PCA involvement is frequently observed in patients with multiple cortical infarctions and resultant mental retardation. The present study provided a unique opportunity to observe the consequences of PCA occlusions in the normalappearing brain. Additional PCA involvement induced a marginal reduction in $\mathrm{CBF}$, suggesting the source of collateral blood supply was exhausted. The WAIS index, as well as its subscores, were significantly lower in patients with PCA involvement than in those without. Regional brain atrophy was also found in the middle cingulum, as was reduced white matter integrity in the left SLF. These findings demonstrate, probably for the first time, the detrimental effects of PCA occlusion from the viewpoints of brain microstructure and anatomical connectivity.

The association among cerebrovascular reactivity, oxygen metabolism, and microstructural impairment in the white matter has been demonstrated previously. ${ }^{24,25}$ We consider nuclear medicine approaches superior to noninvasive MRI measurements such as arterial spin labeling to quantify rCBF, particularly in moyamoya disease. We found correlations between rCBF and FA in the forceps major, as well as an association with fiber tracts such as the SLF. These white matter tracts may be particularly sensitive to cerebral hypoperfusion because the same regions 

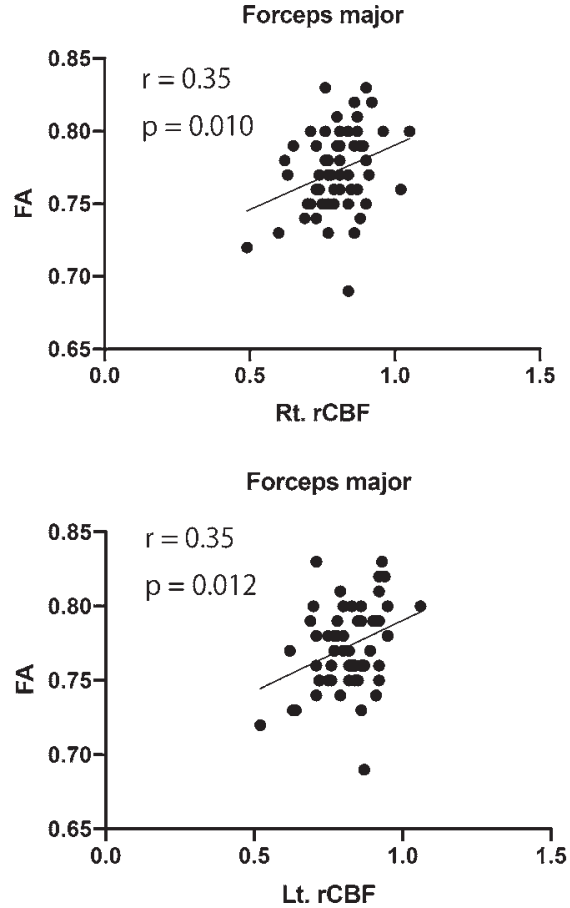

Rt. IFOF

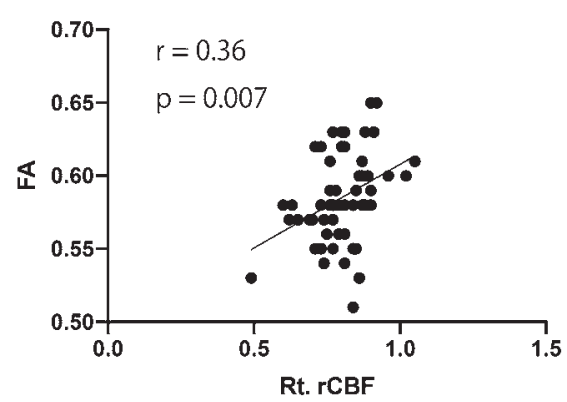

Lt. IFOF

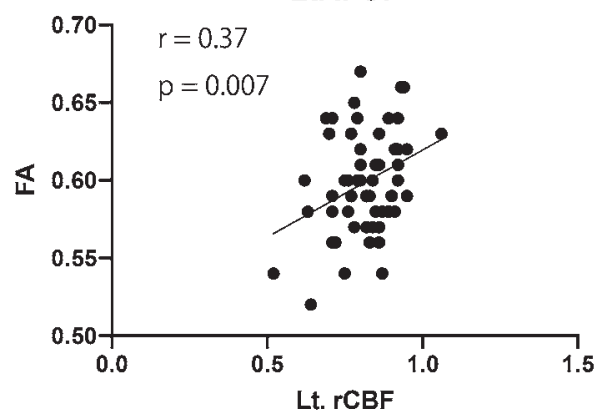

Rt. SLF

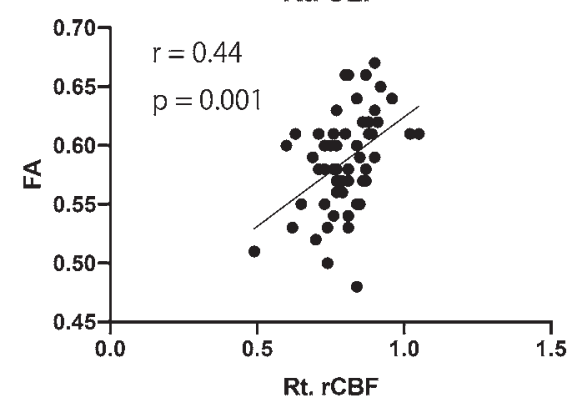

Lt. SLF

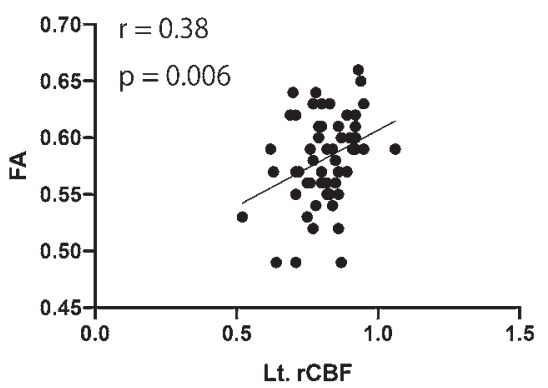

FIG. 5. The scatterplots indicate the correlation between rCBF and FA of the white matter tracts $(r>0.34, p<0.05)$.

are affected in non-moyamoya patients with TIA. ${ }^{26}$ The study of white matter integrity in moyamoya disease has advantages over its study in major cerebral artery disease of arteriosclerotic origin because potential microstructural injuries are probably less influenced by age-related decline. An investigation of white matter integrity in moyamoya disease provided novel insights into the ischemic damage to connections of the association cortex in both developing adolescents and middle-aged individuals.

Various studies have described different mechanisms leading to cognitive impairment in chronic hypoperfusion. ${ }^{3-5}$ Recently, reduced white matter integrity was detected as a common phenomenon in adult patients with moyamoya disease and normal-appearing brain on diffusion MRI. ${ }^{3-5}$ Widespread ischemic damage of the white matter is consistent with findings of an experimental study in which chronic hypoperfusion predominantly affected the white matter. ${ }^{27,28}$ Previous studies have also demonstrated an association between diffusion imaging parameters and cognitive functions. . $^{3,5,11}$ The present study confirmed this relationship between FA derived from DTI and PS. In particular, the FA values of the left SLF were moderately correlated with PS. The SLF is an association fiber tract of the white matter connecting the frontal, occipital, parietal, and temporal lobes. The integrity of the SLF is associated with the PS in young adults, the elderly, individuals with bipolar disorder, and stroke patients. ${ }^{29-31}$ Our results also revealed an association between executive function and global gray matter volume. However, we were not able to identify a localization correlating with executive function; these findings do not fully agree with our previous results. ${ }^{5}$ Some studies have suggested a state of selective neuronal damage in the gray matter involving the central benzodiazepine receptor. ${ }^{32,33}$ Our findings can be interpreted as multiple cortical dysfunctions participating in the task of executive function tests.

Our study may contribute to the improved clinical management of these patients. The implications are important particularly for asymptomatic populations because gradual structural changes, as well as mild cognitive decline, may progress during the follow-up period under conservative treatment. Currently, revascularization surgery in young patients with advanced arterial involvement is a matter of debate when hemodynamic insufficiency is not apparent. ${ }^{34}$ MRI-derived metrics may be used to track early brain damage due to ischemia in such patients. Multimodal MRI metrics can be determined as part of the routine clinical investigation. In the event of ischemic attacks, as well as additional arterial involvement, the suggested approach may be useful in evaluating the consequences and providing information beyond the measurement of $\mathrm{CBF}$ and metabolism. Furthermore, these imaging markers are potentially useful to assess the effectiveness of therapeutic interventions such as revascularization surgery. ${ }^{7}$

\section{Study Limitations}

Our study has potential limitations. First, the rCBF measurement approach may be controversial in its methodology, specifically the sizes and locations of the ROIs. We extracted the $\mathrm{rCBF}$ values from a broad cortical area belonging to the MCA territory because these values represent the gross cerebral hemodynamics. Unfortunately, we were unable to analyze correlations with vasomotor reactivity to acetazolamide because we were not able to use this drug in all study participants. Second, the impact of PCA involvement was investigated in a small population. This may have reduced the statistical power to detect 


\section{A. TMT-A/Gray matter volume}

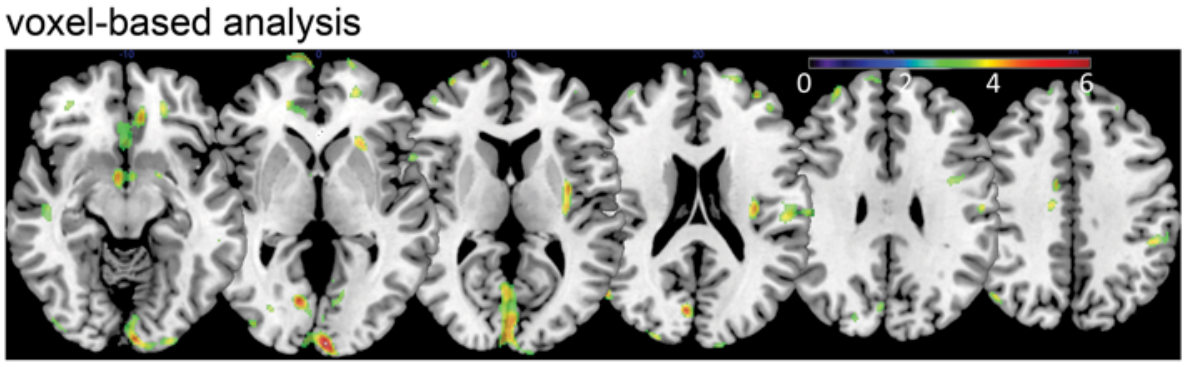

\section{B. PS/FA}

voxel-based analysis
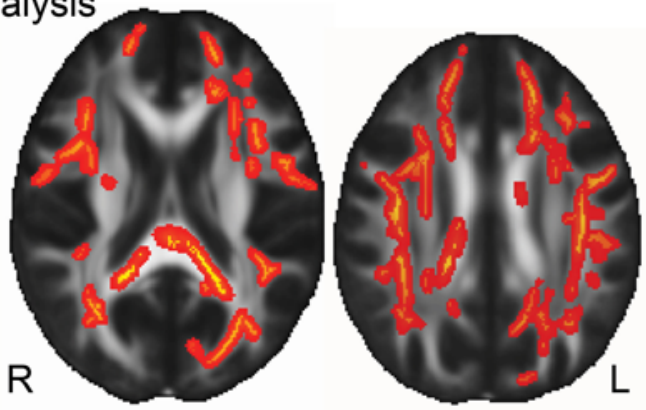

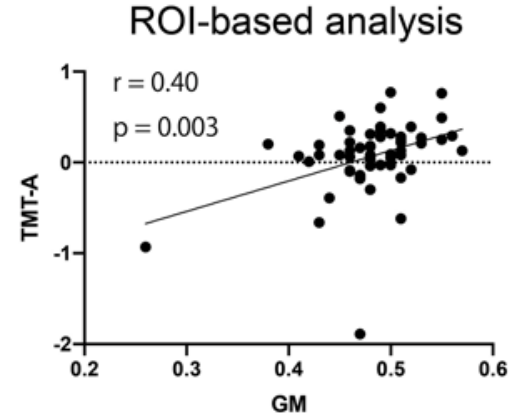

ROI-based analysis

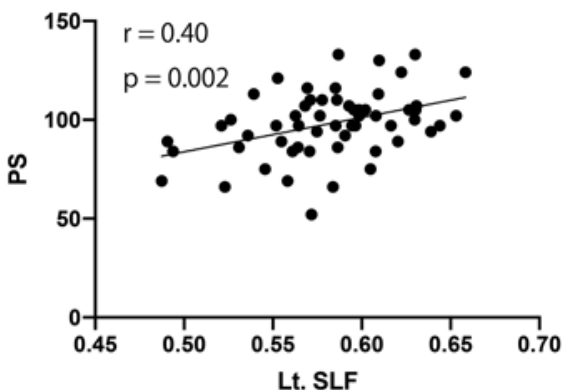

FIG. 6. A: Results of whole-brain voxel-based correlation analyses of GM revealed a moderate positive correlation between TMT part A (TMT-A) and GM volumes in multiple brain regions $(p<0.001$, uncorrected). The scatterplot indicates the correlation between TMT-A and global GM volume $(r=0.40, p=0.003)$. B: The TBSS results showed the association between PS and FA of the white matter $(p<0.05$, TFCE-corrected). The ROI-based analysis revealed the moderate correlation between PS and FA in the left (Lt.) SLF ( $r=0.40, p=0.002)$.

significant changes in both gray and white matter involvement, particularly in the right hemisphere. Third, a longitudinal study would be more optimal to capture changes in microstructural brain damage in relation to disease progression. The present cross-sectional study warrants a study with a longitudinal design to evaluate the impact of disease duration and the association with cerebral perfusion and metabolism.

\section{Conclusions}

Microstructural ischemic brain damage was detected by structural and diffusion MRI in patients with moyamoya disease with no apparent brain lesions. Gray matter volume, particularly in the cingulate gyrus, was associated with advanced arterial involvement and executive function. The parameter FA in association fiber tracts was correlated with advanced arterial involvement, cerebral hypoperfusion, and reduced PS. Thus, combined structural and diffusion MRI is potentially useful for tracking subtle changes in brain structures associated with persistent cerebral hypoperfusion, as well as disease progression.

\section{Acknowledgments}

We gratefully appreciate the financial support of the Research Committee on Moyamoya Disease, sponsored by the Ministry of Health, Labour and Welfare of Japan. Publication fees were covered by a research grant from the Ministry of Health, Labour and Welfare of Japan (Research on Measures for Intractable Diseases). This study was supported by grants for Health and Labour Sciences Research from the Ministry of Health, Labour and Welfare: "Research on Measures for Intractable Disease (Moyamoya Disease)."

\section{References}

1. Gorelick PB, Scuteri A, Black SE, et al. Vascular contributions to cognitive impairment and dementia: a statement for healthcare professionals from the American Heart Association/American Stroke Association. Stroke. 2011;42(9):26722713.

2. Ohta H, Nishikawa H, Kimura H, et al. Chronic cerebral hypoperfusion by permanent internal carotid ligation produces learning impairment without brain damage in rats. Neuroscience. 1997;79(4):1039-1050.

3. Hara S, Hori M, Murata S, et al. Microstructural damage in normal-appearing brain parenchyma and neurocognitive dysfunction in adult moyamoya disease. Stroke. 2018;49(10):2504-2507.

4. Kazumata K, Tha KK, Narita H, et al. Characteristics of diffusional kurtosis in chronic ischemia of adult moyamoya disease: comparing diffusional kurtosis and diffusion tensor imaging. AJNR Am J Neuroradiol. 2016;37(8):1432-1439.

5. Kazumata K, Tha KK, Narita H, et al. Chronic ischemia alters brain microstructural integrity and cognitive performance in adult moyamoya disease. Stroke. 2015;46(2):354360.

6. Su JB, Xi SD, Zhou SY, et al. Microstructural damage pat- 
tern of vascular cognitive impairment: a comparison between moyamoya disease and cerebrovascular atherosclerotic disease. Neural Regen Res. 2019;14(5):858-867.

7. Kazumata K, Tha KK, Tokairin K, et al. Brain structure, connectivity, and cognitive changes following revascularization surgery in adult moyamoya disease. Neurosurgery. 2019;85(5):E943-E952.

8. Kazumata K, Tha KK, Uchino H, et al. Topographic changes in cerebral blood flow and reduced white matter integrity in the first 2 weeks following revascularization surgery in adult moyamoya disease. J Neurosurg. 2017;127(2):260-269.

9. Quon JL, Kim LH, MacEachern SJ, et al. Early diffusion magnetic resonance imaging changes in normal-appearing brain in pediatric moyamoya disease. Neurosurgery. 2020;86(4):530-537.

10. Beaulieu $\mathrm{C}$. The basis of anisotropic water diffusion in the nervous system - a technical review. NMR Biomed. 2002;15(7-8):435-455.

11. Kazumata K, Kamiyama H, Saito H, et al. Direct anastomosis using occipital artery for additional revascularization in moyamoya disease after combined superficial temporal artery-middle cerebral artery and indirect bypass. Oper Neurosurg (Hagerstown). 2017;13(2):213-223.

12. Funaki T, Takahashi JC, Takagi Y, et al. Impact of posterior cerebral artery involvement on long-term clinical and social outcome of pediatric moyamoya disease. J Neurosurg Pediatr. 2013;12(6):626-632.

13. Kazumata K, Tha KK, Uchino H, et al. Mapping altered brain connectivity and its clinical associations in adult moyamoya disease: a resting-state functional MRI study. PLoS One. 2017;12(8):e0182759.

14. Research Committee on the Pathology and Treatment of Spontaneous Occlusion of the Circle of Willis, Health Labour Sciences Research Grant for Research on Measures for Infractable Diseases. Guidelines for diagnosis and treatment of moyamoya disease (spontaneous occlusion of the circle of Willis). Neurol Med Chir (Tokyo). 2012;52:245-266.

15. Houkin K, Nakayama N, Kuroda S, et al. Novel magnetic resonance angiography stage grading for moyamoya disease. Cerebrovasc Dis. 2005;20(5):347-354.

16. Maldjian JA, Laurienti PJ, Burdette JH. Precentral gyrus discrepancy in electronic versions of the Talairach atlas. Neuroimage. 2004;21(1):450-455.

17. Mutsaerts HJ, van Dalen JW, Heijtel DF, et al. Cerebral perfusion measurements in elderly with hypertension using arterial spin labeling. PLoS One. 2015;10(8):e0133717.

18. Ashburner J, Friston KJ. Unified segmentation. Neuroimage. 2005;26(3):839-851.

19. Ashburner J. A fast diffeomorphic image registration algorithm. Neuroimage. 2007;38(1):95-113.

20. Tzourio-Mazoyer N, Landeau B, Papathanassiou D, et al. Automated anatomical labeling of activations in SPM using a macroscopic anatomical parcellation of the MNI MRI singlesubject brain. Neuroimage. 2002;15(1):273-289.

21. Tabesh A, Jensen JH, Ardekani BA, Helpern JA. Estimation of tensors and tensor-derived measures in diffusional kurtosis imaging. Magn Reson Med. 2011;65(3):823-836.

22. Wakana S, Jiang H, Nagae-Poetscher LM, et al. Fiber tractbased atlas of human white matter anatomy. Radiology. 2004;230(1):77-87.

23. Kazumata K, Tokairin K, Sugiyama T, et al. Association of cognitive function with cerebral blood flow in children with moyamoya disease. J Neurosurg Pediatr. 2020;25(1):62-68.

24. Calviere L, Ssi Yan Kai G, Catalaa I, et al. Executive dys- function in adults with moyamoya disease is associated with increased diffusion in frontal white matter. J Neurol Neurosurg Psychiatry. 2012;83(6):591-593.

25. Hara S, Hori M, Ueda R, et al. Unraveling specific brain microstructural damage in moyamoya disease using diffusion magnetic resonance imaging and positron emission tomography. J Stroke Cerebrovasc Dis. 2019;28(4):1113-1125.

26. Ferris JK, Edwards JD, Ma JA, Boyd LA. Changes to white matter microstructure in transient ischemic attack: A longitudinal diffusion tensor imaging study. Hum Brain Mapp. 2017;38(11):5795-5803.

27. Choi BR, Kim DH, Back DB, et al. Characterization of white matter injury in a rat model of chronic cerebral hypoperfusion. Stroke. 2016;47(2):542-547.

28. Wakita $\mathrm{H}$, Tomimoto $\mathrm{H}$, Akiguchi I, et al. Axonal damage and demyelination in the white matter after chronic cerebral hypoperfusion in the rat. Brain Res. 2002;924(1):63-70.

29. Kerchner GA, Racine CA, Hale S, et al. Cognitive processing speed in older adults: relationship with white matter integrity. PLoS One. 2012;7(11):e50425.

30. McKenna BS, Theilmann RJ, Sutherland AN, Eyler LT. Fusing functional MRI and diffusion tensor imaging measures of brain function and structure to predict working memory and processing speed performance among inter-episode bipolar patients. J Int Neuropsychol Soc. 2015;21(5):330-341.

31. Turken A, Whitfield-Gabrieli S, Bammer R, et al. Cognitive processing speed and the structure of white matter pathways: convergent evidence from normal variation and lesion studies. Neuroimage. 2008;42(2):1032-1044.

32. Nakagawara J, Osato T, Kamiyama K, et al. Diagnostic imaging of higher brain dysfunction in patients with adult moyamoya disease using statistical imaging analysis for [123I] iomazenil single photon emission computed tomography. Neurol Med Chir (Tokyo). 2012;52(5):318-326.

33. Yamauchi H, Nishii R, Higashi T, et al. Silent cortical neuronal damage in atherosclerotic disease of the major cerebral arteries. J Cereb Blood Flow Metab. 2011;31(3):953-961.

34. Kuroda S, Ishikawa T, Houkin K, et al. Incidence and clinical features of disease progression in adult moyamoya disease. Stroke. 2005;36(10):2148-2153.

\section{Disclosures}

The authors report no conflict of interest concerning the materials or methods used in this study or the findings specified in this paper.

\section{Author Contributions}

Conception and design: Kazumata. Acquisition of data: Tha. Analysis and interpretation of data: Kazumata, Tokairin, Ito, Uchino, Sugiyama, Kawabori, Tha. Drafting the article: Kazumata. Critically revising the article: Osanai, Tha. Reviewed submitted version of manuscript: Kazumata, Houkin. Approved the final version of the manuscript on behalf of all authors: Kazumata. Statistical analysis: Kazumata. Study supervision: Houkin.

\section{Correspondence}

Ken Kazumata: Hokkaido University Graduate School of Medicine, Sapporo, Japan. kazumata@med.hokudai.ac.jp. 